\title{
The prognostic value of the ratio of standard uptake value of lymph node to primary tumor before treatment of locally advanced nasopharyngeal carcinoma
}

\author{
Yibiao Chen ( $\nabla$ chenyibiao@mzrmyy.com ) \\ Meizhou City People's Hospital \\ Yunlong Lou \\ Meizhou City People's Hospital \\ Dandan Chen \\ Meizhou City People's Hospital \\ zheng lin \\ Meizhou City People's Hospital \\ jianda sun \\ Meizhou City People's Hospital \\ li song \\ Meizhou City People's Hospital \\ wenzhong chen \\ Meizhou City People's Hospital \\ ming zhang \\ Meizhou City People's Hospital
}

\section{Research Article}

Keywords: nasopharyngeal carcinoma, tomography, positron emission type, standard uptake value, Deoxyglucose, Prognosis, Ratio

Posted Date: March 1st, 2022

DOI: https://doi.org/10.21203/rs.3.rs-1340892/v1

License: (c) (1) This work is licensed under a Creative Commons Attribution 4.0 International License. Read Full License 


\section{Abstract}

Background: To evaluate the prognostic value of the ratio of the standard uptake value of the lymph node to the primary tumor before treatment of locally advanced nasopharyngeal carcinoma.

Methods: A total of 180 patients with locally advanced nasopharyngeal carcinoma diagnosed pathologically from January 1, 2016, to December 31, 2018, were selected, and the MEDEX system was used to automatically delineate lymph node metastases SUVmax (LN-SUVmax) and nasopharyngeal carcinoma primary tumor SUVmax (T-SUVmax). In addition, the ratio NTR of LN-SUVmax to T-SUVmx was calculated, and an ROC curve was drawn to obtain the best cut-off value. Kaplan-Meier and Cox regression models were used for survival and multivariate analyses, respectively.

Results: The median follow-up period of 180 patients was 32 (4-62) months. Univariate analysis showed that age $(P=0.013)$, LN-SUVmax $(P=0.001)$, and NTR $(P=0.001)$ were factors influencing overall survival (OS). Factors affecting local progression-free survival (LPFS) were LN-SUVmax $(P=0.005)$ and NTR $(P=0.020)$, while clinical stage $(P=0.023)$, LN-SUVmax $(P=0.007)$, and NTR $(P=0.032)$ were factors affecting Distant metastasis-free survival (DMFS). Multivariate analysis showed that NTR was an independent influencing factor of OS ( $\mathrm{HR}=3.00,95 \% \mathrm{Cl}=1.06-8.4, P=0.038)$, $\mathrm{LPFS}(\mathrm{HR}=3.08,95 \% \mathrm{Cl}=$ 1.27-7.50, $P=0.013)$, and DMFS (HR $=1.84,95 \% \mathrm{Cl}=0.99-3.42, P=0.054)$. Taking OS as the main observation point, the best cut-off point of NTR was 0.95. Kaplan-Meier results showed that the 3-year OS (97.0\% vs. $\left.85.4 \%, c^{2}=11.25, P=0.001\right)$, 3-year LPFS ( $91.3 \%$ vs. $82.1 \%, c^{2}=4.035, P=0.045$ ), and 3year DMFS $\left(92.3 \%\right.$ vs. $\left.87.9 \%, c^{2}=4.576, P=0.032\right)$ of patients with NTR $<0.95$ were higher than those with NTR $>0.95$.

Conclusions: High NTR before treatment may lead to poor prognosis of patients with nasopharyngeal carcinoma. This can serve as a reference value for the reasonable treatment and prognosis monitoring of such patients.

\section{Background}

Nasopharyngeal carcinoma has a high incidence in southern China. Also called Cantonese cancer, it is the most common type of head and neck cancer. However, its cause, treatment, and prognosis are different from other head and neck tumors; the incidence of cervical lymph node and/or distant metastasis is high [1-3]. The high sensitivity of nasopharyngeal carcinoma to radiotherapy and the patient's relatively young age of onset lead to a good overall prognosis. The use of concurrent radiotherapy and chemotherapy for nasopharyngeal carcinoma can achieve a 5-year overall survival (OS) rate and disease-free survival rate of up to $70 \%$ [4]. However, some nasopharyngeal carcinomas relapse or metastasize after treatment, making individualized treatment plans critical. Identifying some prognostic factors that predict treatment outcomes more accurately may help patients benefit from more aggressive treatments. ${ }^{18} \mathrm{~F}$-FDG-PET/CT has been widely used in the diagnosis and staging of patients with nasopharyngeal carcinoma. Many studies have also shown that SUV can be used for risk 
stratification and has good prognostic value [5]. Recently, some studies have shown that the SUV ratio (NTR) of the lymph node to the primary tumor is strongly associated with survival outcome. Hung et al. [6] reported that NTR is an easily available indicator and has a good predictive value for distant metastasis after treatment, which may help determine more personalized treatments or designs in future clinical trials. In the literature, only one study reported on the value of NTR in the survival and prognosis of patients with nasopharyngeal carcinoma. Our study aimed to further examine the prognostic value of NTR in advanced nasopharyngeal carcinoma.

\section{Material And Methods}

\section{Inclusion criteria}

Pathologically diagnosed patients with nasopharyngeal carcinoma; patients who underwent ${ }^{18} \mathrm{~F}$-FDGPET-CT examination before treatment and with complete PET-CT data and clinical data; patients with at least one neck lymph node metastasis and patients without distant metastasis.

\section{Clinical data}

We performed retrospective analysis for 225 patients with nasopharyngeal carcinoma .Among them, 45 patients without lymph node metastasis were excluded, and 180 patients remained (males: 121 (67.2\%); females: 59 (32.8\%))[ Fig. 1]. The age of the patients was 16-82 years (median age, 49.5 years). The pathological types of 179 patients were undifferentiated and differentiated non-keratinizing carcinoma. According to the 2017 UICC or American Joint Committee on Cancer (AJCC) version 8 of the nasopharyngeal carcinoma staging system, there are 27 in T1 stage, 51 in T2 stage, 61 in T3 stage, 41 in T4 stage; 64 in N1 stage, 73 in N2 stage, and 43 in N3 stage. Moreover, there are 24 clinical stages in stage II and 78 in stages III and IV.

\section{Follow-up}

Outpatient and telephone follow-up periods were from the date of diagnosis to April 21, 2021, and the median follow-up time is 32 (4-62) months. After treatment, the frequency of follow-up for the first 3 years was once every 3 months and then every 6 months until the end of follow-up period. Follow-up included routine head and neck examination, nasopharyngeal endoscopy, chest radiograph, and abdominal B-ultrasound. Further examinations were conducted to confirm the diagnosis in case of suspicious problems. The location and time of tumor residue, recurrence, and metastasis were recorded, and rescue treatments (such as re-radiation, surgery, and chemotherapy) were determined according to the severity of the disease. OS was defined as the time from the start of treatment to the death of the patient (for any reason). Local progression-free survival (LPFS) was defined as the time from diagnosis by pathology to the first discovery of local recurrence after treatment or to the last follow-up date. Distant 
metastasis-free survival (DMFS) was defined as the time from the start of treatment until the time when patient had distant metastasis or the time to the last follow-up date.

\section{Treatment}

All patients received radiotherapy, comprising IGRT and VMAT. The chemotherapy regimen in concurrent radiotherapy and chemotherapy was platinum single drugs (cisplatin, nedaplatin, and carboplatin), and the neoadjuvant chemotherapy regimen or adjuvant chemotherapy regimen was platinum combination regimen (paclitaxel combined with platinum, paclitaxel + fluorouracil + platinum, and fluorouracil combined with platinum).

\section{PET/CT imaging}

Imaging instrument was Biograph mCT.s20 from Siemens, Germany ( 16 rows CT). ${ }^{18} \mathrm{~F}-\mathrm{FDG}$ was provided by the Guangzhou Isotope Center of the Chinese Academy of Atomic Energy, with a radiochemical purity of $95 \%$. Before the PET/CT examination, all patients were fasted for more than 4 hours. The blood glucose was measured before the examination, and the blood glucose was controlled below $11 \mathrm{mmol} / \mathrm{L}$. ${ }^{18} \mathrm{~F}-\mathrm{FDG} 5.55 \mathrm{MBq} / \mathrm{kg}$ was injected intravenously according to body weight, and patients were rested for 50-60 minutes until PET/CT imaging. The scan range was from the upper middle of the thigh to the top of the skull. The CT scan parameters were as follows: voltage, $140 \mathrm{kV}$; current, $110 \mathrm{~mA}$; tube single-turn rotation time, $0.5 \mathrm{~s}$; and layer thickness, $3 \mathrm{~mm}$. The PET scan duration was $3 \mathrm{~min}$ per bed, and the PET image attenuation was corrected based on the CT image.

\section{Image analysis}

The PET and CT images are sent to the MMWP22510 workstation for image fusion and image processing analysis. The region of interest (ROI) was delineated automatically with the $40 \%$ threshold of SUVmax, and the optimal boundary of tumor was determined by manual adjustment in axial position. The primary tumor and lymph node SUVmax were automatically delineated and acquired by MEDEX software. The maximal SUV in each region of interest was determined using the whole body attenuation corrected image [Figure 2]. The maximum SUV (SUVmax) was defined as the highest activity concentration per injected dose per body weight after a correction for radioactive decay. The T-SUVmax and LN-SUVmax were defined as the SUVmax at the primary tumor and the SUVmax of the highest neck lymph node metastasis, respectively.

\section{Statistical analysis}

SPSS software was used to draw ROC curve and OS was used as the main observation point to obtain the boundary value of SUVmax of nasopharyngeal carcinoma primary tumor and metastasis. Among 
them, the parameter value corresponding to the maximum Youden index was defined as the grouping boundary point. Youden index $=$ (sensitivity + specificity) -1 . The Kaplan-Meier method was used to calculate OS, LPFS, and DMFS using SPSS21.0 software, the Log-rank method for testing and univariate prognostic analysis, and the Cox regression model for multivariate prognostic analysis. $P<0.05$ indicated significant difference.

\section{Results}

A total of 180 patients with nasopharyngeal carcinoma were included in the analysis. The median survival time of OS, LPFS, and DMFS were 60 months, 55 months, and 55 months, respectively. The rates of 3-year OS, 3-year LPFS, and 3-year DMFS were $91 \%, 93 \%$, and $91 \%$, respectively. Among the 180 patients, 11 had fatal events, 13 had recurrence and metastasis, 2 had simple local recurrence in the nasopharynx, 5 had simple neck lymph node recurrence, 1 had nasopharyngeal and neck recurrence, and 2 had local recurrence accompanied by distant metastasis. Distant metastases occurred in 12 patients: 2 liver metastases, 8 lung metastases, 6 bone metastases, 1 mediastinal metastasis, and 1 abdominal lymph node metastasis.

Factors analyzed in the study: General clinical factors and PET-related parameters, including age, gender, T stage, N stage, clinical stage, pathological type, T-SUVmax, LN-SUVmax, and NTR.

Determination of the cut-off value of each parameter: With OS as the main survival end point, the boundary value of each parameter was determined according to the ROC curve. The best cut-off value of NTR related to OS was 0.95 (AUC of $0.710, P=0.044,95 \% \mathrm{Cl}=0.560-0.860$, sensitivity $=0.750$, and specificity $=0.674)($ Figure 3$)$. The patients were divided into two groups: the high NTR group (NTR > $0.95 ; n=80)$ and the low NTR group (NTR $<0.95 ; n=100)$. The cut-off values of the other two parameters were T-SUVmax of 12.35 (AUC $=0.582, P=0.361,95 \% \mathrm{Cl}=0.395-0.770$, sensitivity $=0.636$, specificity $=$ 0.586 ) and LN-SUVmax of 9.32 (AUC $=0.765, P=0.003,95 \% \mathrm{Cl}=0.650-0.879$, sensitivity $=0.909$, specificity $=0.521$ ). A total of 93 patients had LN-SUVmax $>9.32$ and 87 had LN-SUVmax $<9.32$. The results of univariate prognostic analysis of different survival endpoints are shown in Table 1. A univariate analysis of OS, LPFS, and DMFS of the whole group of patients was carried out. The observation targets were OS mainly, LPFS, and DMFS. Univariate analysis showed that age, LN-SUVmax, and NTR were associated with OS $(P=0.013,0.001$, and 0.001 , respectively); LN-SUVmax and NTR with LPFS $(P=$ 0.005 and 0.020 , respectively), and clinical stage, LN-SUVmax, and NTR with DMFS $(P=0.023,0.007$, and 0.032 , respectively). Kaplan-Meier results showed that the 3 -year OS $\left(97.0 \%\right.$ vs. $85.4 \%, c^{2}=11.25, P=$ $0.001)$, 3-year LPFS (91.3\% vs. $\left.82.1 \%, c^{2}=4.035, P=0.045\right)$, and 3 -year DMFS $\left(92.3 \%\right.$ vs. $87.9 \%, c^{2}=$ 4.576, $P=0.032$ ) of patients with NTR $<0.95$ were higher than those with NTR $>0.95$ (Figure 4-8). In addition, patients with LN-SUVmax $<9.32$ had better 3-year OS (100\% vs. $\left.88.0 \%, c^{2}=10.565, P=0.001\right)$, 3-year LPFS ( $96.6 \%$ vs. $\left.93.4 \%, c^{2}=7.715, P=0.005\right)$, and 3-year DMFS (97.8\% vs. $82.5 \%, c^{2}=7.387, P=$ $0.007)$ than patients with LN-SUVmax $>9.32$. 
Multivariate analysis showed that NTR was a significant influencing factor for OS ( $\mathrm{HR}=3.00,95 \% \mathrm{Cl}=$ $1.06-8.48, P=0.038)$ and LPFS $(\mathrm{HR}=3.08,95 \% \mathrm{Cl}=1.27-7.50, P=0.013)$, while only marginally

significant for DMFS ( $\mathrm{HR}=1.84,95 \% \mathrm{Cl}=0.99-3.42, P=0.054)$ Table 2. In addition, $\mathrm{N}$ stage $(\mathrm{HR}=3.32$, $95 \% \mathrm{Cl}=1.04-10.66, P=0.044)$ and clinical stage $(\mathrm{HR}=9.78,95 \% \mathrm{Cl}=1.91-50.15, P=0.006)$ were also influencing factors of LPFS, while clinical stage was a marginally significant influencing factor of DMFS $(\mathrm{HR}=2.12,95 \% \mathrm{Cl}=0.99-4.55, P=0.053)$.

\section{Table 1}

Univariate prognostic analysis of different survival endpoints in 180 patients with nasopharyngeal carcinoma 


\begin{tabular}{|c|c|c|c|c|c|c|c|}
\hline Variables & & os & & & LPFS & & DMFS \\
\hline & $\mathbf{n}$ & $c^{2}$ & $P$ value & c2 & $P$ value & $c^{2}$ & $P$ value \\
\hline Sex & & 3.009 & 0.083 & 3.817 & 0.051 & 2.949 & 0.086 \\
\hline male & 121 & & & & & & \\
\hline female & 59 & & & & & & \\
\hline Age & & 6.119 & 0.013 & 3.733 & 0.053 & 3.682 & 0.055 \\
\hline$>59.5$ & 41 & & & & & & \\
\hline$<59.5$ & 139 & & & & & & \\
\hline T stage & & 0.064 & 0.800 & 1.712 & 0.191 & 3.646 & 0.056 \\
\hline T1 & 27 & & & & & & \\
\hline T2 & 51 & & & & & & \\
\hline T3 & 61 & & & & & & \\
\hline T4 & 41 & & & & & & \\
\hline N stage & & 3.425 & 0.064 & 0.545 & 0.460 & 1.857 & 0.173 \\
\hline N1 & 64 & & & & & & \\
\hline N2 & 73 & & & & & & \\
\hline N3 & 43 & & & & & & \\
\hline Clinical stage & & 3.011 & 0.083 & 0.758 & 0.384 & 5.175 & 0.023 \\
\hline ॥ & 24 & & & & & & \\
\hline III & 78 & & & & & & \\
\hline IV & 43 & & & & & & \\
\hline Pathological type & & 0.088 & 0.767 & 0.088 & 0.767 & 0.049 & 0.824 \\
\hline $\begin{array}{l}\text { Undifferentiated keratinizing } \\
\text { carcinoma }\end{array}$ & 179 & & & & & & \\
\hline $\begin{array}{l}\text { Differentiated non-keratinizing } \\
\text { carcinoma }\end{array}$ & 1 & & & & & & \\
\hline T-SUVmax & & 1.877 & 0.171 & 0.049 & 0.825 & 0.076 & 0.783 \\
\hline$<12.35$ & 103 & & & & & & \\
\hline
\end{tabular}




\begin{tabular}{|lccccccc|}
\hline$>12.35$ & 77 & & & & & & \\
\hline LN-SUVmax & & 10.565 & 0.001 & 7.715 & 0.005 & 7.387 & 0.007 \\
\hline$<9.32$ & 87 & & & & & & \\
$>9.32$ & 93 & & & & & & \\
\hline NTR & & 11.25 & 0.001 & 5.385 & 0.020 & 4.576 & 0.032 \\
\hline$<0.95$ & 100 & & & & & & \\
$>0.95$ & 80 & & & & & & \\
\hline
\end{tabular}

Abbreviation T-SUVmax: SUVmax of primary nasopharyngeal carcinoma, LN-SUVmax: SUVmax of lymph node metastases, NTR: ratio of LN-SUVmax to T-SUVmax

\section{Table 2}

Cox multivariate analysis 


\begin{tabular}{|c|c|c|c|c|c|c|}
\hline & OS & & LPFS & & DMFS & \\
\hline & $\mathrm{HR}(95 \% \mathrm{Cl})$ & $P$ & $\mathrm{HR}(95 \% \mathrm{Cl})$ & $P$ & $\mathrm{HR}(95 \% \mathrm{Cl})$ & $P$ \\
\hline $\begin{array}{l}\text { Sex } \\
\text { male vs female }\end{array}$ & & & & & & \\
\hline $\begin{array}{l}\text { Age } \\
>59.5 \text { vs }<59.5\end{array}$ & & & & & $\begin{array}{l}1.74 \rrbracket 1.01- \\
3.01 \rrbracket\end{array}$ & 0.046 \\
\hline $\begin{array}{l}\text { T stage } \\
\mathrm{T} 1+\mathrm{T} 2 \text { vs } \mathrm{T} 3+\mathrm{T} 4\end{array}$ & & & & & & \\
\hline $\begin{array}{l}\text { N stage } \\
\mathrm{N} 1+\mathrm{N} 2 \text { vs N3 }\end{array}$ & & & $\begin{array}{l}3.32 \otimes 1.04- \\
10.66 \rrbracket\end{array}$ & 0.044 & & \\
\hline $\begin{array}{l}\text { Clinical stage } \\
\text { II+III vs IV }\end{array}$ & & & $\begin{array}{l}9.78 \otimes 1.91- \\
50.15 \rrbracket\end{array}$ & 0.006 & $\begin{array}{l}2.12 \varangle 0.99- \\
4.55 \rrbracket\end{array}$ & 0.053 \\
\hline T-SUVmax & & & & & & \\
\hline $\begin{array}{l}<12.35 \text { vs } \\
>12.35\end{array}$ & & & & & & \\
\hline $\begin{array}{l}\text { LN-SUVmax } \\
<9.32 \text { vs }>9.32\end{array}$ & & & & & & \\
\hline $\begin{array}{l}\text { NTR } \\
<0.95 \text { vs }>0.95\end{array}$ & $\begin{array}{l}3.00(1.06- \\
8.48)\end{array}$ & 0.038 & $3.08(1.27-7.50)$ & 0.013 & $\begin{array}{l}1.84 \rrbracket 0.99- \\
3.42 \rrbracket\end{array}$ & 0.054 \\
\hline
\end{tabular}

Abbreviation T-SUVmax: SUVmax of primary nasopharyngeal carcinoma, LN-SUVmax: SUVmax of lymph node metastases, NTR: ratio of LN-SUVmax to T-SUVmax.,HR:hazard ratio,Cl,:confidence interval.

\section{Discussion}

The prognostic factors of malignant tumors have always been the focus of clinical attention, because these help develop and determine more targeted treatments. Southern China has the highest incidence of nasopharyngeal carcinoma, with an annual incidence of 20-50 cases per 100,000 people. According to the 7th AJCC staging system, $60-70 \%$ of patients have locally advanced nasopharyngeal carcinoma (LANPC) $[2,3]$. With the continuous development of radiotherapy techniques such as intensity-modulated radiotherapy and concurrent radiotherapy and chemotherapy, the regional control rate of nasopharyngeal carcinoma reaches $90 \%$, while distant metastasis is the main reason of treatment failure. Therefore, to improve the prognosis and perform personalized treatments, patients with advanced nasopharyngeal carcinoma should be classified according to the risk of metastasis $[5,7,8]$. Recent studies have shown that MTV and TLG are better than SUV alone in predicting prognosis. Compared with MTV or SUV, TLG is 
expected to provide better prognostic stratification because it theoretically integrates tumor volume and glucose metabolism. Wang et al. [9] found that SUVmax and TLG are the influencing factors of 3-year LRFS and 3-year FFS through single factor analysis, while SUVmax is the only independent influencing factor through multifactor analysis. Sdeng et al. [10] found that PET-related parameters are related to the prognosis of patients with nasopharyngeal carcinoma, among which MTV has a strong correlation with OS, EFS, and LRC. Yoon et al. [11] found that among the metabolic parameters of PET, TLG is the most useful prognostic factor for PFS, OS, LRFFS, and DFFS, and these results are consistent with the finding of Moon et al. [12], which showed that TLG of the primary tumor is a good metabolic prognostic factor in patients with nasopharyngeal carcinoma undergoing concurrent radiotherapy and chemotherapy. Jin et al. $[13,23]$ believed that SUVmax-N is an independent prognostic factor in determining patients with locally advanced nasopharyngeal carcinoma. Furthermore, combining SUVmax-N with clinical staging could influence the level of risk of locally advanced patients, thereby changing treatment decisions, and improving patient survival. Our study showed that lymph node SUVmax was a prognostic factor of OS, LPFS, and DFFS in univariate analysis, but it was not an independent factor in multivariate analysis.

Several recent studies have studied the clinical significance of NTR in different tumors, including esophageal cancer, breast cancer, and lung cancer and explored the role of NTR in the assessment of lymph node metastasis. Cerfolio et al. [14,22] reported that an NTR of 0.56 is the best cut-off value for predicting mediastinal lymph node metastasis in patients with non-small cell lung cancer, and its value is significantly better than that of the primary tumor SUVmax; Park et al. [15] also confirmed that in breast cancer, NTR could better predict the metastasis of axillary lymph nodes than SUVmax in the lymph node with the highest axillary metabolism. Several studies have further explored the role of NTR in clinical prognosis. Kaira et al. [16] found that in non-small cell lung cancer, high NTR has worse PFS and OS than low NTR, and it did not respond well to initial therapy. Similarly, Kim HR [17], Kim YH [18], and Chung $[19,20]$ also confirmed that NTR is an independent influencing factors of predicting of cervical squamous cell carcinoma, endometrial carcinoma, invasive ductal breast cancer, and resectable pancreatic cancer.

So far, there is only one related report on the application of NTR in nasopharyngeal carcinoma, which showed the prognostic value of NTR in nasopharyngeal carcinoma DMFS. Hung et al. [6] showed that NTR is an independent influencing factor of DMFS in nasopharyngeal carcinoma. However, there was only one survival endpoint of DMFS that was analyzed, and there are no relevant reports on the prognosis of other survival endpoints. Our study is the first to report the value of NTR in OS and LPFS of nasopharyngeal carcinoma. Chen [1] mentioned that induction chemotherapy before concurrent radiotherapy and chemotherapy is a good treatment strategy, because this method can improve the survival of patients with nasopharyngeal carcinoma and the control rate of distant metastasis. However, the eligibility of patients for induction chemotherapy needs further research. In the present study, we found that the 3-year OS, 3-year LPFS, and 3-year DMFS of patients with NTR $<0.95$ were higher than those with NTR $>0.945(P=0.001, P=0.045$ and $P=0.032)$, and NTR was the independent factor for predicting OS and LPFS, suggesting that the metabolic activity of metastatic lymph node SUVmax relative to the primary tumor might be a promising prognostic indicator for patients with nasopharyngeal carcinoma. Moreover, our study also found that NTR was an independent influencing factor of DMFS in 
nasopharyngeal carcinoma, which was consistent with the results of Hung et al. [6]. This conclusion also suggested that in future work, we should pay attention to the relative metabolic activity of lymph nodes and primary tumors. For those with a high ratio of these two, appropriate additional radiation doses could be added during treatment to increase the tumor LPRS rate and prolong the patient's OS, reducing the rate of distant metastasis. As this study aimed to investigate whether the relative metabolic activity of lymph nodes and primary tumors had prognostic value in locally advanced nasopharyngeal carcinoma, we found that NTR obtained by preprocessing was an independent predictor of OS and LC. Therefore, we believe that NTR might become a valuable prognostic indicator, allowing patients to benefit from more aggressive treatment. Our univariate analysis showed that LN-SUVmax was an important factor in predicting recurrence, while multivariate analysis did not. The non-standardized SUV used in different studies might be the reason for this difference. Moreover, the patient's tumor type, blood sugar level, the interval between imaging agent injection time and scanning time, machine type, collection time per bed, and other factors could affect the scanning effect, leading to SUV variability. Different from previous studies, our study added another parameter NTR, the ratio of SUVmax of lymph node metastases to SUVmax of the primary tumor, to predict OS, LPFS, and DMFS of advanced nasopharyngeal carcinoma. NTR could also reflect the biological interaction between the primary tumor of nasopharyngeal carcinoma and metastatic lymph nodes. A recent study by Park et al. [21] showed that in breast cancer, the ratio of SUV of axillary metastatic lymph nodes to the SUV of the primary tumor is more accurate than the SUVmax of axillary metastatic lymph nodes to determine the rate of lymph node metastasis involvement.

The main limitation of this study was that it was a retrospective study conducted in a single center. Different chemotherapy regimens could affect the results. Furthermore, although our hospital had a standardized plan for PET scanning to minimize possible variations between scanners, biological and technical variations would still exist, which might lead to selection bias, thereby limiting the universality of our findings. Further verification of the prognostic value requires prospective and large-scale research on NTR before it can be widely used in clinical practice. Nevertheless, the present study is still worthy of attention because it is the first study to report NTR in the prognosis of locally advanced nasopharyngeal carcinoma. Our findings may serve as a reference for further research on the importance of metabolic activity related to metastatic lymphatic pretreatment.

\section{Conclusion}

In summary, high NTR before treatment may lead to poor prognosis of patients with nasopharyngeal carcinoma. This can serve as a reference value for the reasonable treatment and prognosis monitoring of such patients.

\section{Abbreviations}

NPC: Nasopharyngeal carcinoma SUV: Maximum standardized uptake value; LN-SUVmax; lymph node metastases SUVmax; T-SUVmax: primary tumor SUVmax; NTR : the ratio of LN-SUVmax to TSUVmx; ROC: Analysis receiver operating characteristic analysis; AUC: Area-under-curve; Cl: Confidence 
interval; CRT: Chemoradio Therapy ; OS: Overall survival; DMFS: Distant metastasis-free survival; LPFS: Local progression -free survival; FDG PET/CT: 2-deoxy-2- ${ }^{18} \mathrm{~F}$ fluoro-D-glucose positron emission tomography/computed tomography; HR: Hazard ratio; AJCC: American Joint Committee on Cancer ; UICC :Union for International Committee on Cancer ; IGRT: Image Guided Radiation Therapy; VMAT: Volumetric Modulated ArcTherapy; MTV: Metabolic tumor volume; TLG: Total lesion glycolysis; PFS: Progression-free survival.

\section{Declarations}

\section{Acknowledgements}

We would like to thank Wei Lin and Quan Li for the data acquisition.We also thank Xingxiang Zhong ,Zimin Huang and Zhifen Qiu for their excellent technical support.

\section{Authors' contributions}

Yun long Lou and Dandan Chen: data analysis and manuscript writing, editing. Zheng Lin, Jianda Sun, Li Song, Wenzhong Chen, Ming Zhang and Yibiao Chen initiated and designed the study. All authors read and approved the final manuscript..We declare that this manuscript is original, has not been published before and is not currently being considered for publication elsewhere.

\section{Availability of data and materials}

The datasets generated during and/or analyzed during the current study are available from the corresponding author on reasonable request.

\section{Ethics approval and consent to participate}

This study was approved by the ethics committee of Meizhou people's hospital.All patients signed an informed consent approved by the institutional Review Board. The images in the article have been approved by the patients. All the authors have followed the applicable ethical standards to maintain the research integrity without any duplication, fraud or plagiarism issues.

\section{Competing interests}

The authors declare that they have no competing interests. 


\section{References}

1. ChenYP, Chan ATC, Le QT, Blanchard $P$,et al .Nasopharyngeal carcinoma.Lancet 2019;394:64-80

2. Torre LA, Bray F, Siegel RL, et al. Global cancer statistics.CA Cancer J Clin 2015; 65: 87-108.

3. Edge SB and Compton CC, et al. The American Joint Committee on Cancer: the 7th edition of the AJCC cancer staging manual and the future of TNM. Ann Surg Oncol 2010; 17: 1471-1474.

4. Liu X, Li X, Jiang N, et al. Prognostic value of chronic hepatitis B virus infection in patients with nasopharyngeal carcinoma: analysis of 1301 patients from an endemic area in China. Cancer 2014; 120(1): 68-76.

5. Li WF, Sun Y, Mao YP, et al. Proposed lymph node staging system using the International Consensus Guidelines for lymph node levels is predictive for nasopharyngeal carcinoma patients from endemic areas treated with intensity modulated radiation therapy. Int J Radiat Oncol Biol Phys 2013; 86: 249256.

6. Tsung-Min Hunga , Kang-Hsing Fana, Chung-Jan Kang.J,et al Lymph node-to-primary tumor standardized uptake value ratio on PET predicts distant metastasis in nasopharyngeal carcinoma.Oral Oncology2020,110:1368-8375.

7. Xiao WW, Huang SM, Han F, et al. Local control, survival, and late toxicities of locally advanced nasopharyngeal carcinoma treated by simultaneous modulated accelerated radiotherapy combined with cisplatin concurrent chemotherapy: long-term results of a phase 2 study. Cancer 2011; 117(9): 1874-1883.

8. Ng WT, Lee MC, Hung WM, et al. Clinical outcomes and patterns of failure after intensity-modulated radiotherapy for nasopharyngeal carcinoma. Int J Radiat Oncol Biol Phys 2011; 79: 420-428

9. Cheng tao Wang ouyang b, zhang $t$ et al. Study on the influence of ${ }^{18}$ FDG PET/CT parameters on the prognosis of nasopharyngeal carcinoma [J]. Chinese journal of radiation oncology, 2016,3(25):207211.

10. Zschaeck S, Li Y, Lin Q, Beck M,Amthauer H, Bauersachs L, et al.Prognostic value of baseline 18Ffluorodeoxyglucose positron emission tomography parameters MTV, TLG and asphericity in aninternational multicenter cohort of nasopharyngeal carcinoma patients.PLoS ONE 2020,15(7): e0236841.

11. Hong In Yoon,Kyung Hwan Kim, Jeongshim Lee,et al. The Clinical Usefulness of 18FFluorodeoxyglucose Positron Emission Tomography to Predict Oncologic Outcomes and PET-Based Radiotherapeutic Considerations in Locally Advanced Nasopharyngeal Carcinoma .Cancer Res Treat.2016;48(3):928-941.

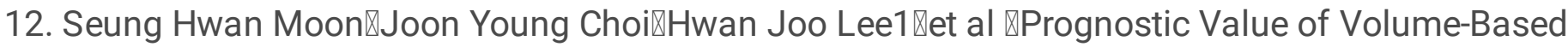
Positron Emission TomographyComputed Tomography in Patients WithNasopharyngeal Carcinoma Treated With Concurrent Chemoradiotherapy Clinical and Experimental Otorhinolaryngology June $2015,8(2): 142-148$. 
13. Ya-Nan Jin, Ji-Jin Yao, Si-Yang Wang, Wang-Jian Zhang, et al.Prognostic value of primary gross tumor volume and standardized uptake value of ${ }^{18} \mathrm{~F}-\mathrm{FDG}$ in PET/CT for distant metastasis in locoregionally advanced nasopharyngeal carcinoma. Tumor Biology July 2017: 1-7.

14. Cerfolio, R.J, Bryant, A.S, et al. Ratio of the maximum standardized uptake value on FDG-PET of the mediastinal lymph nodes to the primary tumor may be a universal predictor of nodal malignancy in patients with nonsmall-cell lung cancer. Ann. Thorac. Surg. 2007, 83, 1826-1829.

15. Park J, Byun BH, Noh WC, Lee SS, Kim HA, Kim EK, et al. Lymph node to primary tumor SUV ratio by ${ }^{18} \mathrm{~F}-\mathrm{FDG} \mathrm{PET} / \mathrm{CT}$ and the prediction of axillary lymph node metastases in breast cancer. Clin Nucl Med 2014;39:e249-53.

16. Kaira, K, Endo, M, Asakura, K,et al. Ratio of standardized uptake value on PET helps predict response and outcome after chemotherapy in advanced non-small cell lung cancer. Ann. Nucl. Med. 2010, 24, 697-705.

17. Kim, H.R, Seo, M, Nah, Y.W,et al. Clinical impact of ${ }^{18}$-ffluorodeoxyglucose positron emission tomography/computed tomography in patients with resectable pancreatic cancer: diagnosing lymph node metastasis and predicting survival. Nucl. Med. Commun. 2018, 39, 691-698.

18. Kim, Y.H, Yoon, H.J, Kim, Y, et al. Axillary Lymph Node-to-Primary Tumor Standard Uptake Value Ratio on Preoperative ${ }^{18} \mathrm{~F}-\mathrm{FDG}$ PET/CT: A Prognostic Factor for Invasive Ductal Breast Cancer. J. Breast Cancer 2015,18, 173-180.

19. Chung, H.H, Cheon, G.J, Kim, J.W, Park, N.H,et al. Prognostic value of lymph node-to-primary tumor standardized uptake value ratio in endometrioid endometrial carcinoma. Eur. J. Nucl. Med. Mol. Imaging 2018,45, 47-55.

20. Chung, H.H,Cheon, G.J, Kim, J.W,et al. Prognostic importance of lymph node-to-primary tumor standardized uptake value ratio in invasive squamous cell carcinoma of uterine cervix.Eur.J. Nucl. Med. Mol. Imaging 2017, 44, 1862-1869.

21. Park, J, Byun, B.H, Noh, W.C, Lee, S.S et al. Lymph node to primary tumor SUV ratio by 18F-FDG PET/CT and the prediction of axillary lymph node metastases in breast cancer. Clin. Nucl. Med. 2014, 39, e249-e253.

22. Mattes, M.D, Moshchinsky, A.B, Ahsanuddin, S,Rizk, N.Pet al.Ratio of Lymph Node to Primary Tumor SUV on PET/CT Accurately Predicts Nodal Malignancy in Non-Small-Cell Lung Cancer. Clin. Lung Cancer 2015, 16, e253-e258.

23. Jeong Y, Baek S, Park JW, Joo JH, Kim JS, Lee S-Wet, et al. Lymph node standardized uptake values at pre-treatment $18 \mathrm{~F}$-fluorodeoxyglucose positron emission tomography as a valuable prognostic factor for distant metastasis in nasopharyngeal carcinoma. Br J Radiol 2017; 90: 20160239.

\section{Figures}


Searrch for patients with biopsy-proven, newly diagnosed NPC who underwent PET scan between 2016 and 2018

101 patients with distant metastasis at diagnosis or a history of other malignancies and received any treatment before ${ }^{18} \mathrm{~F}-\mathrm{FDG} \mathrm{PET} / \mathrm{CT}$ scan were excluded

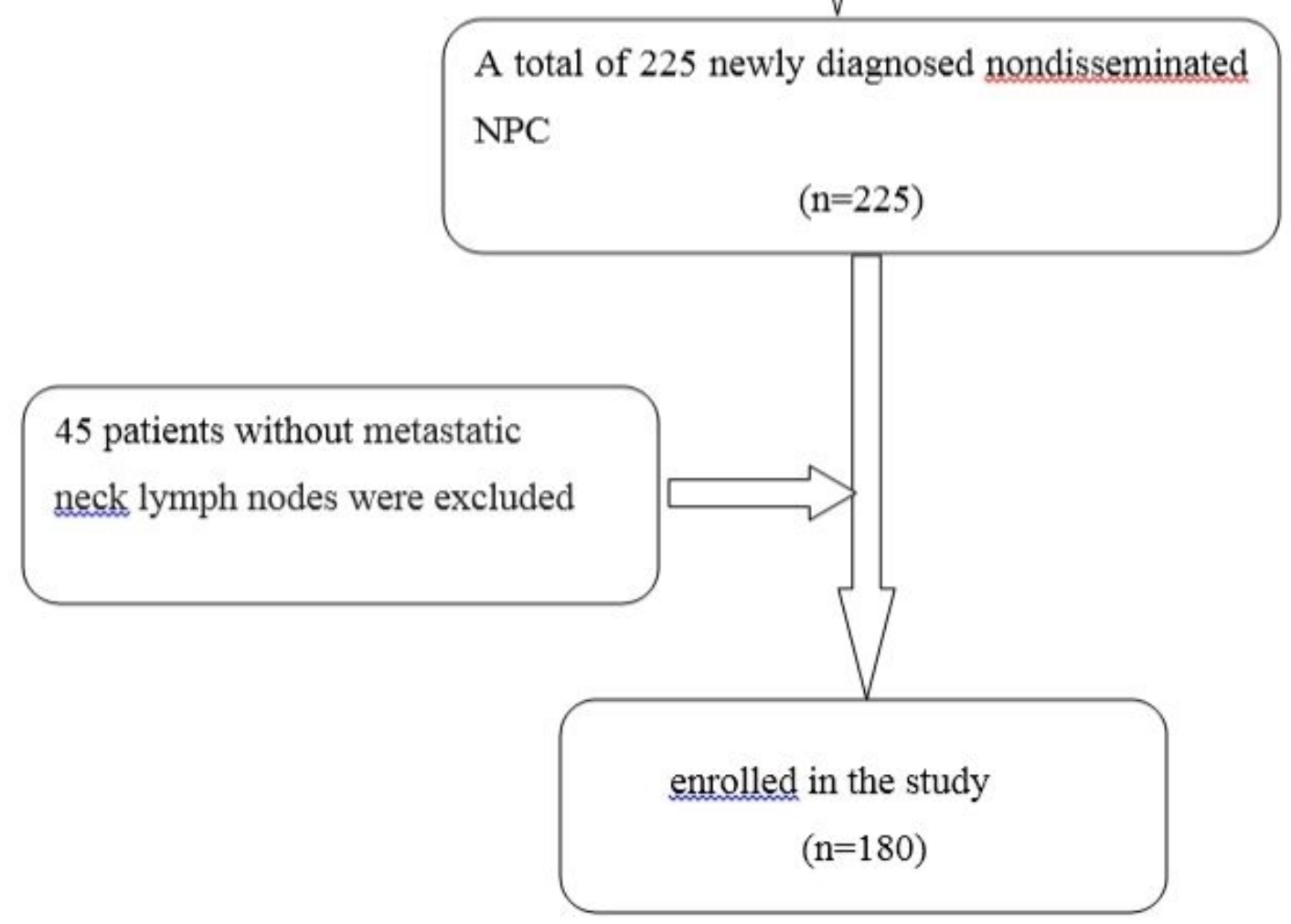

\section{Figure 1}

Flowchart of the nasopharyngeal carcinoma patient's cohort 


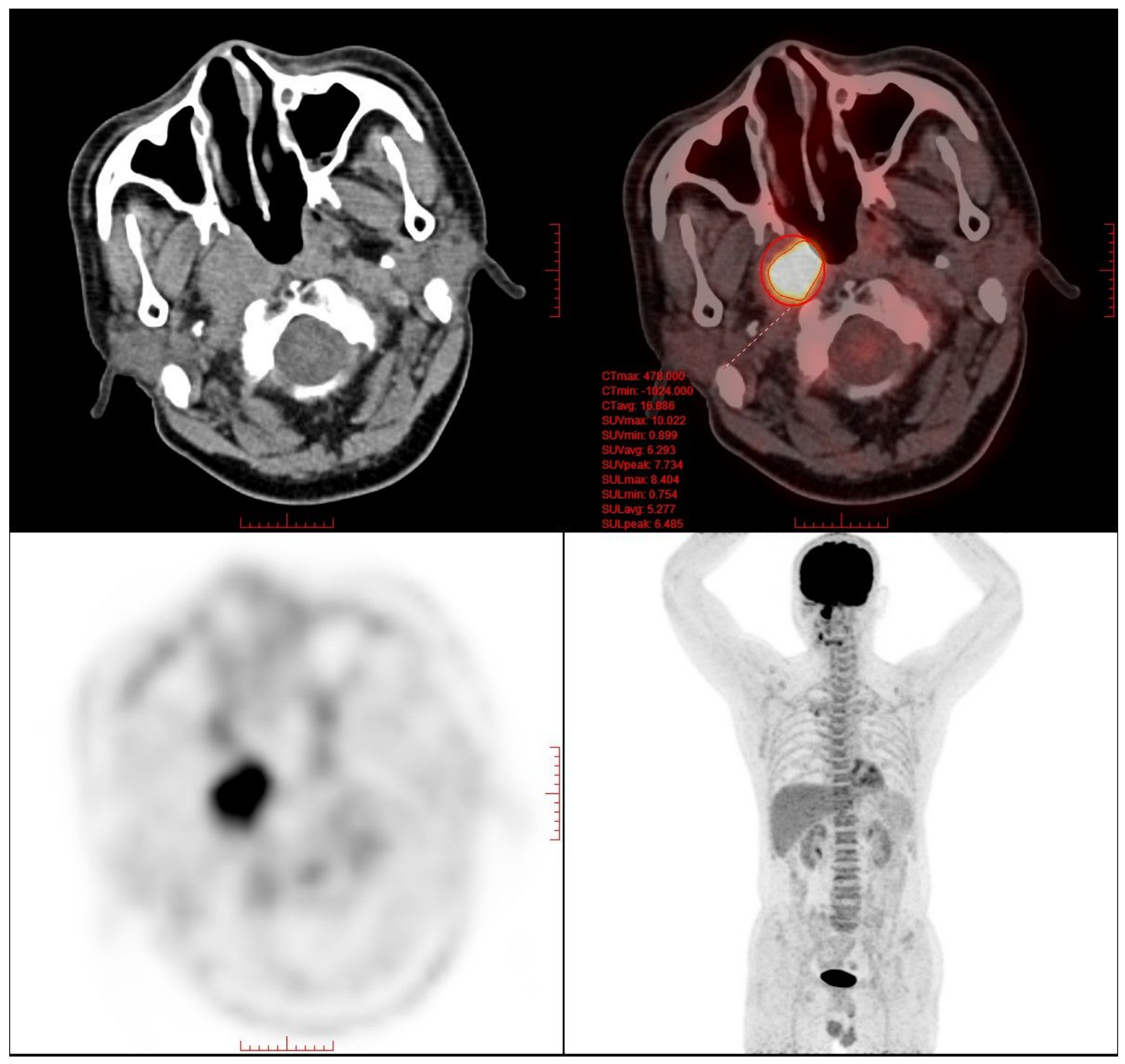

Figure 2

A 56-year-old male patient with nasopharyngeal cancer.The maxium standardized uptake value were determined by drawing a region of interest around the primary tumor on the transaxial slices 


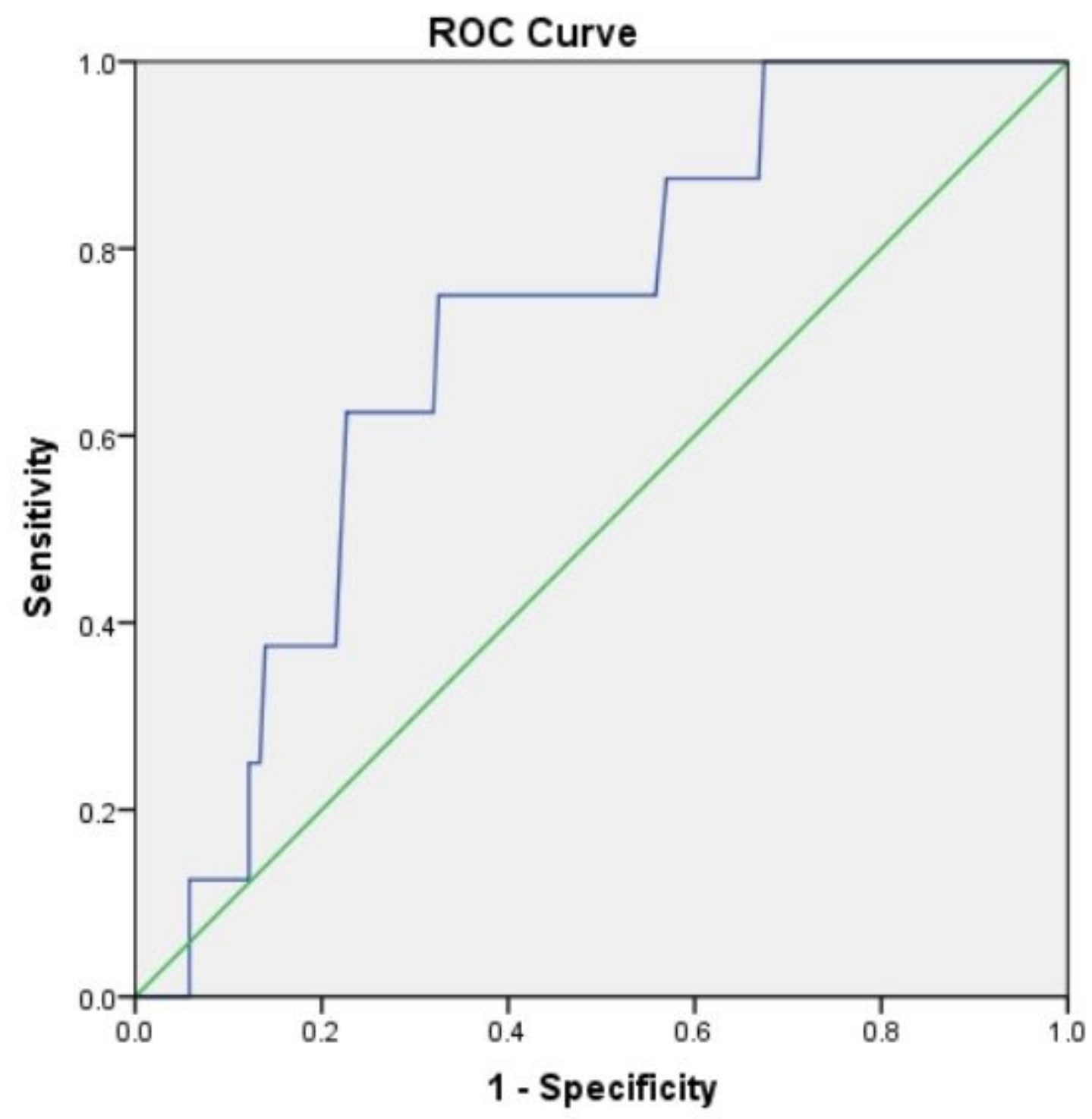

Figure 3

ROC curve analysis of OS based on NTR value, AUC under the curve is $0.710,(P=0.044,95 \% \mathrm{Cl}$ of $0.560-$ $0.860)$, and the best cut-off value is 0.95 


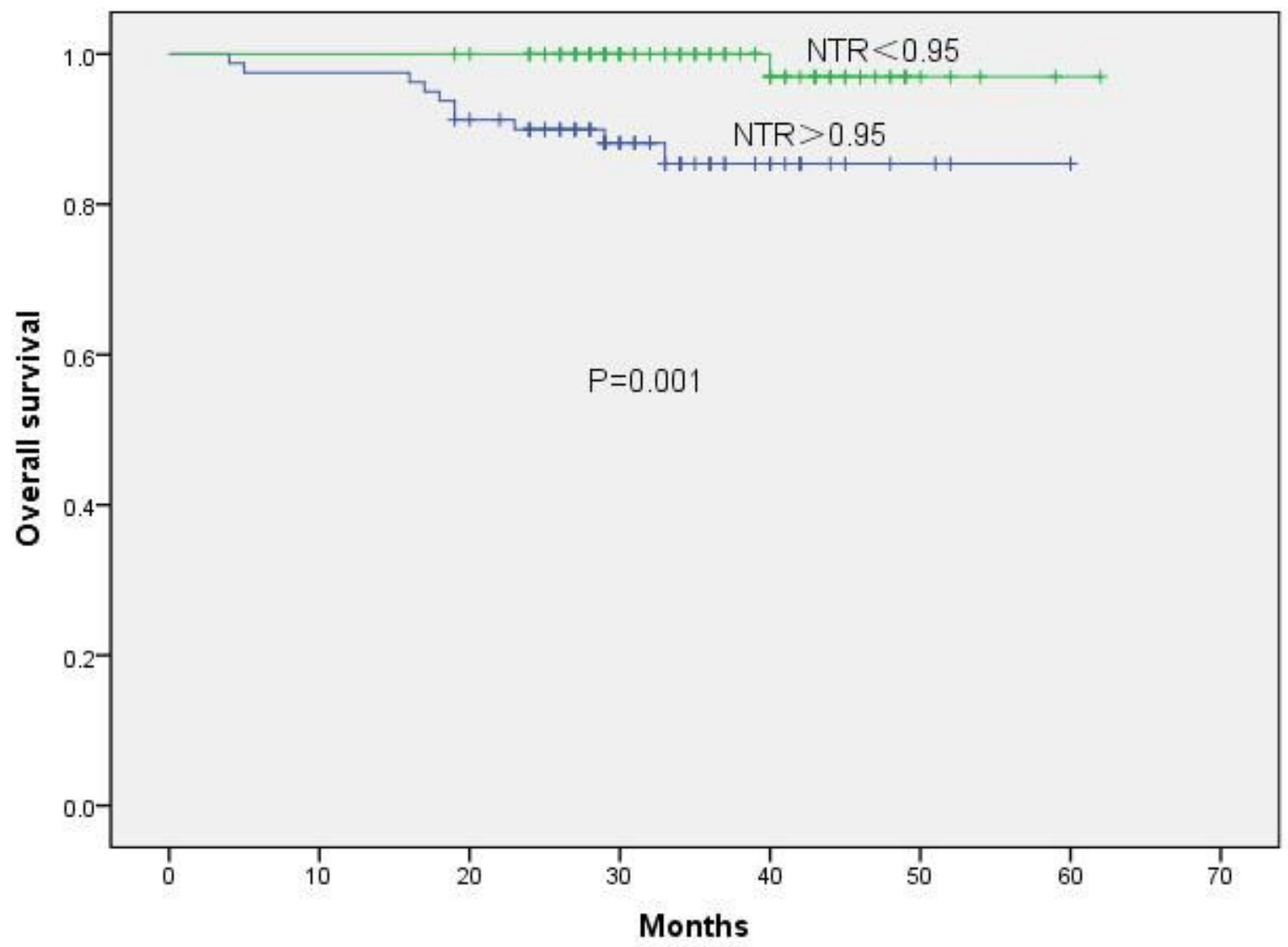

Figure 4

Kaplan-Meier survival curve of OS, stratified according to NTR, $P=0.001$ 


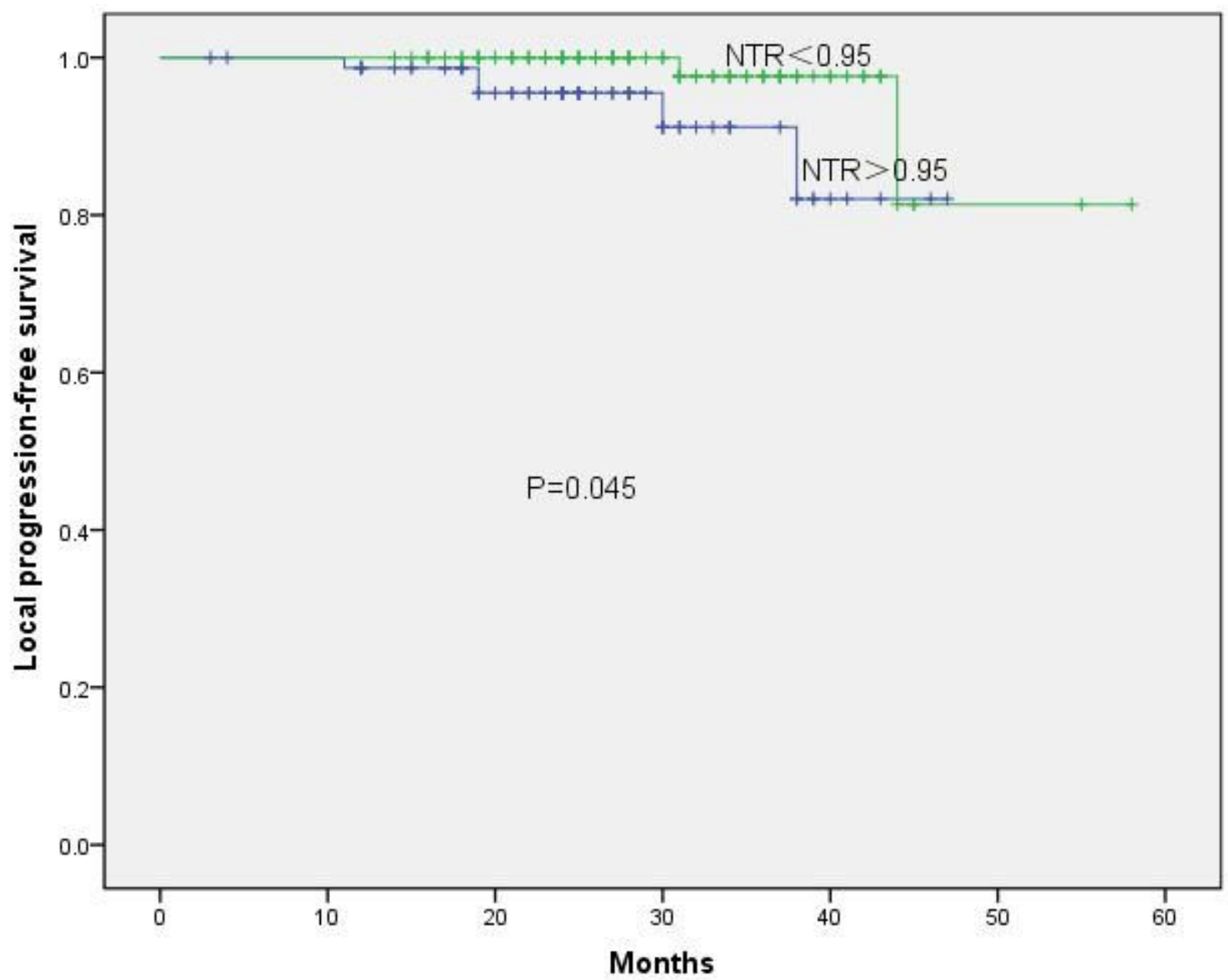

Figure 5

Kaplan-Meier survival curve of LPFS, stratified according to NTR, $P=0.045$ 


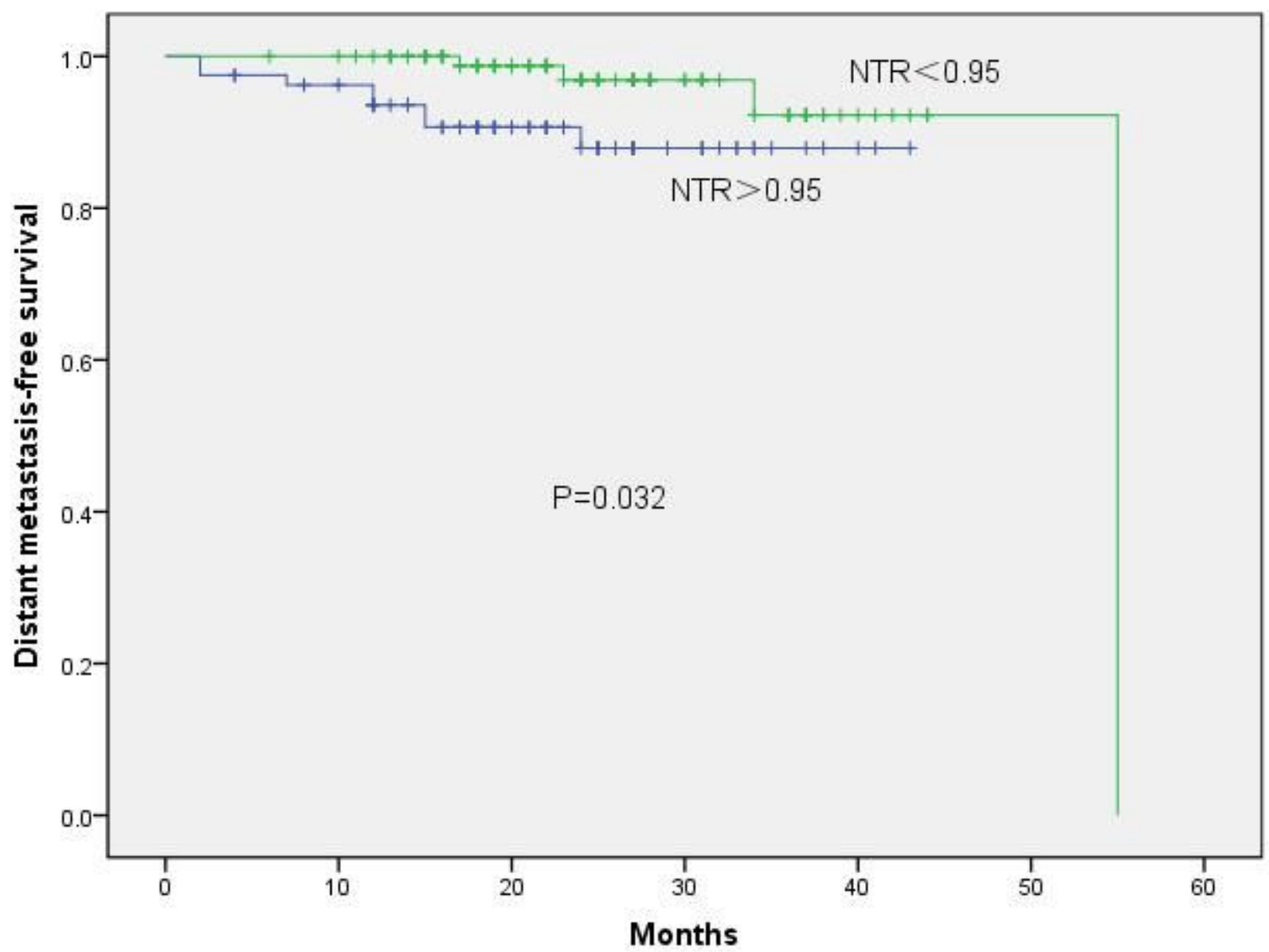

Figure 6

Kaplan-Meier survival curve of DMFS, stratified according to NTR, $P=0.032$ 

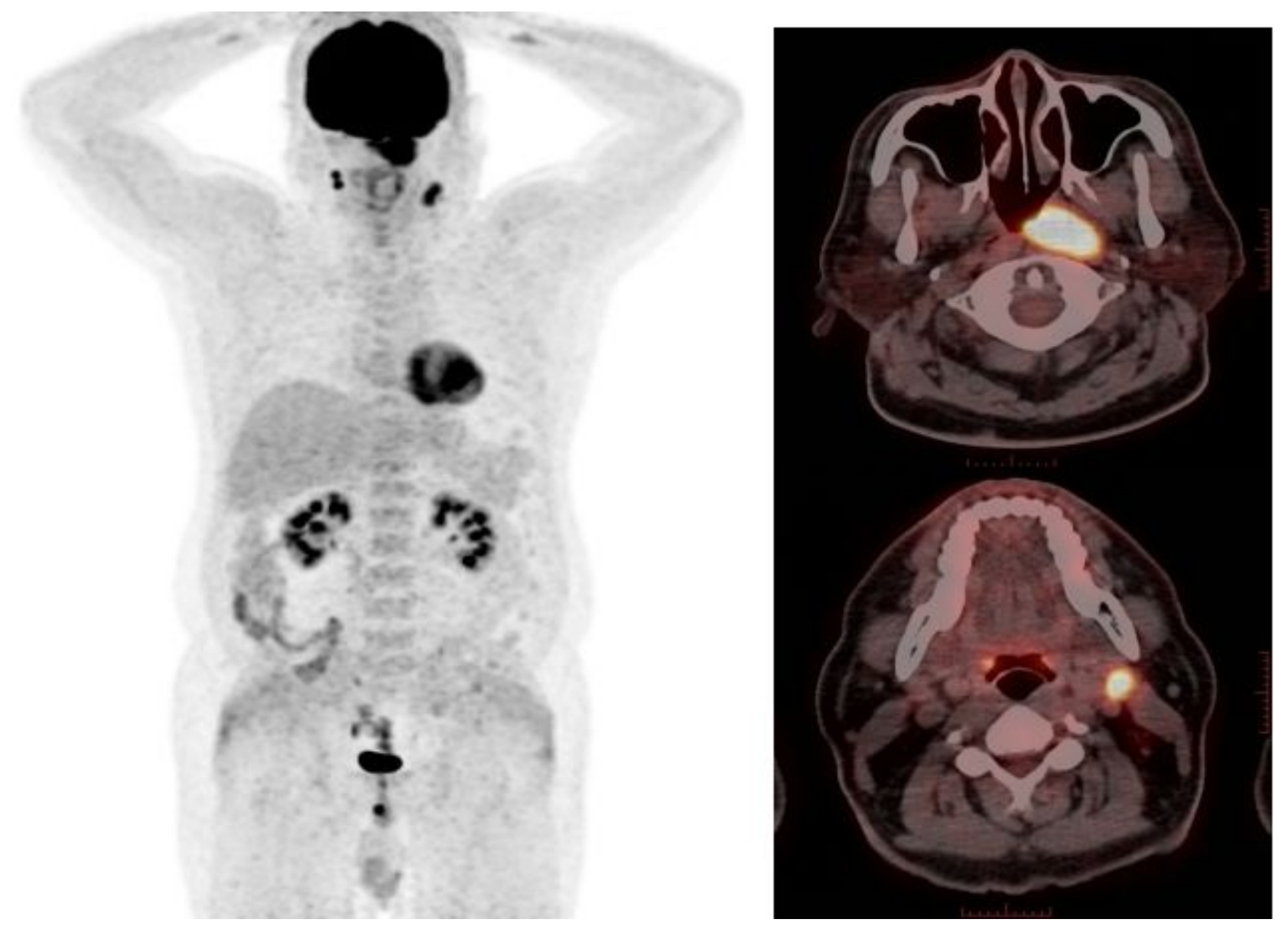

\section{Figure 7}

Transaxial fused images and MIP images of ${ }^{18} \mathrm{~F}-\mathrm{FDG}$ PET/CT of a 56 -year-old man with stage III (T2N2M0) nasopharyngeal carcinoma. The primary tumor (red arrow) was arising from the left nasopharyngeal recess (A), T-SUVmax11.78. The lymph node with the highest ${ }^{18} \mathrm{~F}$-FDG uptake (white arrow) was in the left left neck level Ila (B), LN-SUVmax7.31. The NTR was 0.66. The OS ,LRFS and DMFS were 26,24 and24 months, respectively. 

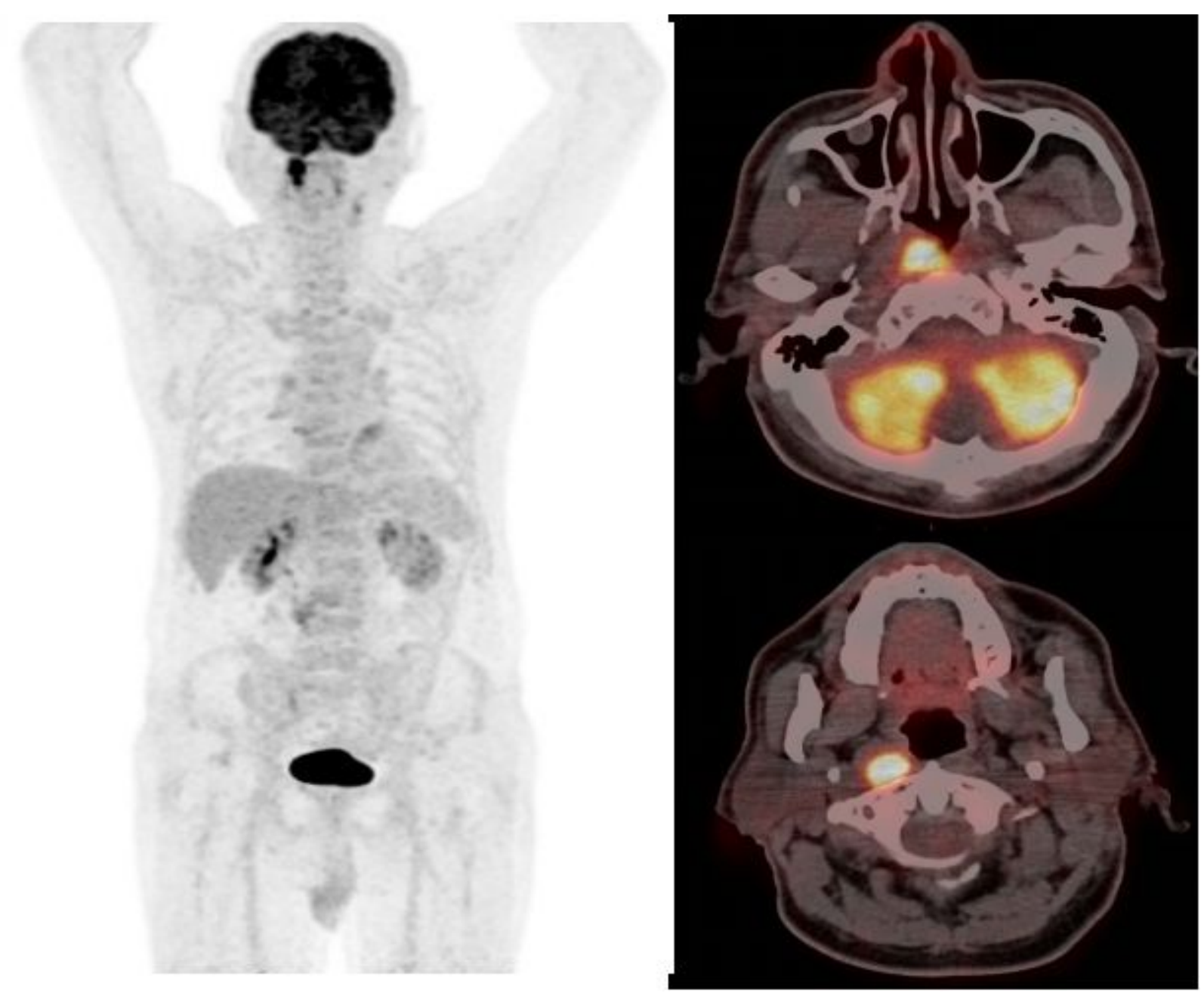

\section{Figure 8}

Transaxial fused images and MIP images of ${ }^{18} \mathrm{~F}$-FDG PET/CT of a 68 -year-old man with stage III (T2N1M0) nasopharyngeal carcinoma. The primary tumor (red arrow) was arising from the right nasopharyngeal recess (A), T-SUVmax9.86. The lymph node with the highest ${ }^{18} \mathrm{~F}$-FDG uptake (white arrow) was the right retropharyngeal space (B), LN-SUVmax12.56. The NTR was1.27. The OS ,LRFS and DMFS were 20,12 and17 months, respectively. 